Original Article

\title{
Benefits of short-term structured exercise in non-overweight women with polycystic ovary syndrome: a prospective randomized controlled study
}

\author{
Volkan Turan ${ }^{1)}$, Ebru Kaya Mutlu' ${ }^{2)}$, Ulas Solmaz ${ }^{1)}$, Atalay Ekin ${ }^{1)^{*}}$, Ozge Tosun ${ }^{3)}$, Gokhan \\ Tosun $^{1)}$, Emre Mat ${ }^{1)}$, Cenk Gezer ${ }^{1)}$, Mehtap Malkoc ${ }^{3)}$ \\ 1) Department of Obstetrics and Gynecology, Tepecik Training and Research Hospital: Izmir, Turkey \\ 2) Department of Physiotherapy and Rehabilitation, Faculty of Health Sciences, Istanbul University, \\ Turkey \\ 3) Department of Physiotherapy, Dokuz Eylul University, Turkey
}

\begin{abstract}
Purpose] The short-term effects of structured exercise on the anthropometric, cardiovascular, and metabolic parameters of non-overweight women diagnosed with polycystic ovary syndrome were evaluated. [Subjects and Methods] Thirty women with a diagnosis of polycystic ovary syndrome were prospectively randomized to either a control group $(n=16)$ or a training group $(n=14)$ for a period of 8 weeks. Anthropometric, cardiovascular, and metabolic parameters and hormone levels were measured and compared before and after the intervention. [Results] Waist and hip measurements (anthropometric parameters); diastolic blood pressure; respiratory rate (cardiovascular parameters); levels of low-density lipoprotein cholesterol, total cholesterol, fasting glucose, and fasting insulin; and the homeostasis model assessment of insulin resistance index (metabolic parameters) were significantly lower in the training group after 8 weeks of exercise compared to the baseline values. After exercise, the training group had significantly higher oxygen consumption and high-density lipoprotein levels and significantly shorter menstrual cycle intervals. The corresponding values for controls did not significantly differ between the start and end of the 8-week experiment. [Conclusion] Short-term regular exercise programs can lead to improvements in anthropometric, cardiovascular, and metabolic parameters of non-overweight women with polycystic ovary syndrome.

Key words: Exercise, Non-overweight, Polycystic ovary syndrome
\end{abstract}

(This article was submitted Feb. 25, 2015, and was accepted Apr. 16, 2015)

\section{INTRODUCTION}

Polycystic ovary syndrome (PCOS) occurs in 5-10\% of women of reproductive age ${ }^{1)}$. Chronic anovulation, hyperandrogenism, and insulin resistance are the main characteristics of $\mathrm{PCOS}^{2)}$. These patients also have elevated triglyceride and low-density lipoprotein (LDL) cholesterol levels and low high-density lipoprotein (HDL) cholesterol levels ${ }^{3}$.

Treatment is generally focused on normalizing anovulation and reducing metabolic syndrome parameters. Diet and exercise are recommended as first-line treatments by the majority of gynecologists and endocrinologists. The aim is to reduce abdominal fat, hyperandrogenemia, and insulin resistance and to improve lipid profiles ${ }^{4}$. Published studies have demonstrated the positive effects of exercise training on maximal oxygen consumption $\left(\mathrm{MaxVO}_{2}\right)$, weight, and

*Corresponding author. Atalay Ekin (E-mail: atalayekin@ hotmail.com)

(C)2015 The Society of Physical Therapy Science. Published by IPEC Inc. This is an open-access article distributed under the terms of the Creative Commons Attribution Non-Commercial No Derivatives (by-ncnd) License $<$ http://creativecommons.org/licenses/by-nc-nd/3.0/>. waist circumferences in PCOS patients ${ }^{5)}$. Combined aerobic and resistance exercise is more effective than either aerobic or resistance exercise alone in improving insulin sensitivity and glycemic control and in reducing abdominal fat in obese women with $\mathrm{PCOS}^{6,7)}$. However, the metabolic and clinical characteristics of PCOS patients who are not overweight, and thus the efficacy of the various forms of exercise, have not been evaluated ${ }^{8}$. For example, in overweight PCOS patients, exercise programs lasting 12 weeks tended to yield positive results ${ }^{5,9)}$, whereas benefits might be achieved more quickly by PCOS patients who are not overweight.

We hypothesized that 8 weeks of structured exercise would be sufficient to improve the anthropometric, cardiovascular, and metabolic parameters of non-overweight women with PCOS, at least over the short term. Therefore, we conducted a randomized controlled clinical trial to establish the beneficial effects of exercise on these 3 sets of parameters in non-overweight PCOS patients.

\section{SUBJECTS AND METHODS}

We evaluated PCOS patients whose body mass index (BMI) was in the normal range $\left(<25 \mathrm{~kg} / \mathrm{m}^{2}\right)$ according to the definition of obesity for Asians. All patients were admit- 
ted to our center between March 2011 and May 2014. The study was approved by the Human Research Ethics Committee of Dokuz Eylul University, Izmir, Turkey (reference no.: 03/38-09, GOA385). Written informed consent was obtained from all patients. PCOS patients were diagnosed on the basis of Rotterdam Criteria (2003), which requires the presence of two of the following: ${ }^{10)}$ (1) a polycystic ovary, defined as the presence of $>10$ cysts $2-8 \mathrm{~mm}$ in diameter, an ovarian volume $>10 \mathrm{~cm}^{3}$, and an echodense stroma on transvaginal or pelvic ultrasonography; ${ }^{11)}(2)$ clinical hyperandrogenism (Ferriman-Gallwey ${ }^{12}$ ) score $>8$ ) or biochemical hyperandrogenism (serum testosterone level $>3.6 \mathrm{pg} / \mathrm{mL}$ in the absence of other causes of hyperandrogenism); and (3) oligomenorrhea and/or anovulation.

The following patients were excluded: patients with endocrinological diseases, including diabetes, thyroid, adrenal, or pituitary gland dysfunction; cardiovascular, hepatic, or pulmonary disease; a history of orthopedic or other physical symptoms that would otherwise limit exercise performance; and those who had exercised regularly within the last 6 months. The resulting 32 non-overweight women with PCOS were randomly allocated into a training group $(\mathrm{n}=$ 16) that participated in a structured exercise program for 8 weeks or a control group $(n=16)$ that did not participate in a structured exercise program. The trial flow chart is shown in Fig. 1. Randomization was carried out using a computergenerated random number table and pre-labeled, sealed envelopes. Based on the results, participants were assigned to one of the two groups.

Anthropometric parameters (body mass index, waist, and hip measurements), cardiovascular parameters (systolic and diastolic blood pressure, heart rate, respiratory rate, Max$\mathrm{VO}_{2}$ ), metabolic parameters (HDL cholesterol, LDL cholesterol, triglycerides, total cholesterol, fasting glucose, fasting insulin, and homeostasis model assessment [HOMA] insulin resistance index), and hormone levels (follicle stimulating hormone $[\mathrm{FSH}]$, luteinizing hormone $[\mathrm{LH}]$, estradiol, total testosterone, and free testosterone) were measured pre- and post-intervention. The dates of the patients' menstrual cycles were also recorded. All clinical assessments were performed by the same physician.

Blood samples were obtained between 7:30 a.m. and 8:30 a.m., (after overnight fasting) during the early follicular phase (days 2-5) of a progesterone-induced menstrual cycle. Blood samples were obtained from supine patients after a 30 -min resting period and collected in tubes containing ethylenediaminetetraacetic acid. The samples were immediately centrifuged at $4{ }^{\circ} \mathrm{C}$ for $20 \mathrm{~min}$ at $1,600 \mathrm{~g}$ and stored at $-20{ }^{\circ} \mathrm{C}$ until assayed. Plasma LH, FSH, estradiol, and free and total testosterone levels were measured using specific radioimmunoassay. Blood insulin and glucose levels were measured with a solid-phase chemiluminescent enzyme immunoassay and the glucose oxidase method, respectively. Insulin resistance was estimated based on the HOMA index (basal glucose $\mathrm{mmol} \times$ basal insulin $\mathrm{mIU} / \mathrm{mL} / 22.5$ ).

Hip and waist circumferences were determined using a tape measure, measuring the widest circumference of the buttocks and the smallest circumference of the waist. Blood pressure was measured with a manual sphygmomanometer from a seated patient after a 5-min resting period. Heart and

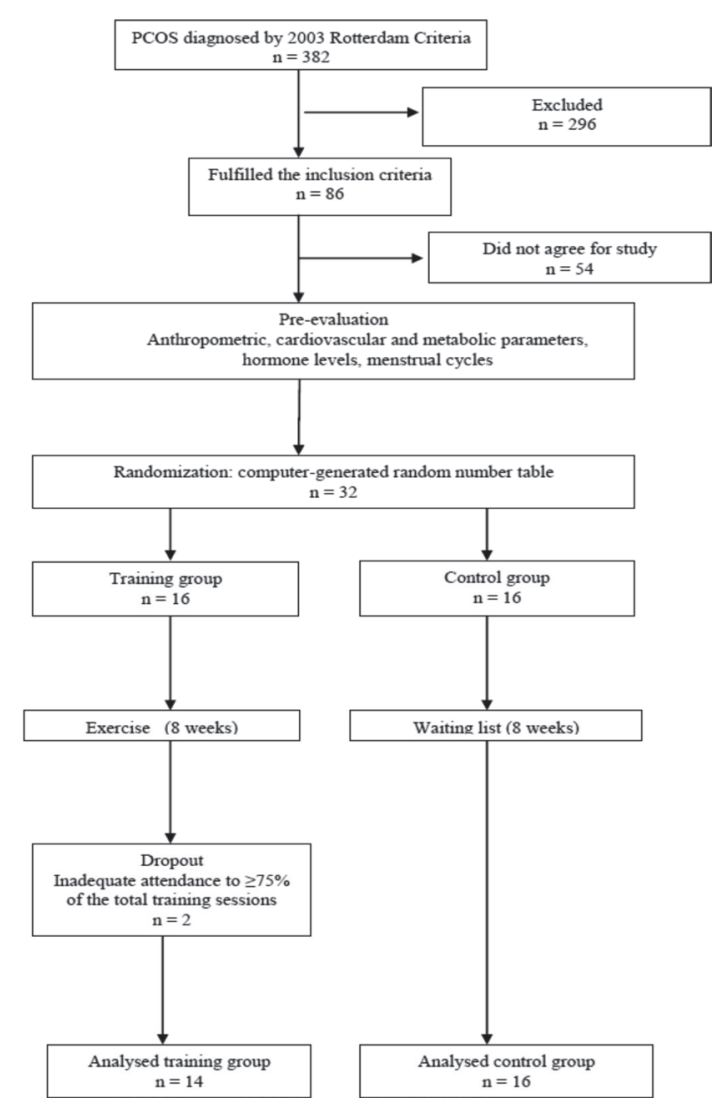

Fig. 1. Study flow chart

respiratory rates were evaluated manually. The radial pulse was measured over a period of $10 \mathrm{~s}$. $\mathrm{MaxVO}_{2}$ was calculated indirectly during a 6 -min walking test ${ }^{13}$ ) performed as follows: patients were given a stopwatch and asked to begin walking on a flat track at their natural walking speed. At the end of $6 \mathrm{~min}$, the walking distance in meters was recorded. Heart rate, blood pressure, and respiratory rate were recorded before and after the test. The following formula was used to calculate $\mathrm{MaxVO}_{2}$ at the end of the 6-min walking test:

$\mathrm{MaxVO}_{2}=132.853-(0.0769 \times$ weight $)-(0.3877 \times$ age $)$ $+(6.315 \times$ gender $($ male: 1 , female: 0$)-(3.2649 \times$ time $)-$ $(0.1565 \times$ heart rate $)$.

At the beginning of the study, general dietary and behavioral advice, but not a structured calorie restriction program, was provided to all study participants. All patients were counseled regarding a healthy, balanced meal plan with regular food and a nutritional composition in which $50 \%$ of the calories were from carbohydrates, $25 \%$ from protein, and $25 \%$ from fat.

Patients participated in a structured exercise program 3 times per week for 8 weeks at the Physical Therapy and Rehabilitation Fitness Unit of Dokuz Eylul University. During each session (50-60 min), the patients performed aerobic and resistance exercises. Each session was preceded by a 5-min warm-up and static stretching of the upper and lower limbs, neck, and trunk muscles, followed by a 5-min cool down. The training sessions were supervised by a physiotherapist. Weekly physical activity was initially monitored 
Table 1. Comparison of anthropometric and cardiovascular parameters between and within groups

\begin{tabular}{lcccccc}
\hline & \multicolumn{3}{c}{ Training group $(\mathrm{n}=14)$} & \multicolumn{3}{c}{ Control group $(\mathrm{n}=16)$} \\
\hline Variable & Baseline & $8 \mathrm{w}$ later & Difference & Baseline & 8 w later & Difference \\
\hline BMI $\left(\mathrm{kg} / \mathrm{m}^{2}\right)$ & $21.8 \pm 1.0$ & $21.7 \pm 1.1$ & $0.1 \pm 0.1$ & $21.9 \pm 1.1$ & $21.7 \pm 1.1$ & $0.04 \pm 0.1$ \\
Waist measurement $(\mathrm{cm})$ & $68.8 \pm 2.1$ & $67.6 \pm 1.9$ & $1.18 \pm 0.4^{*}$ & $68.4 \pm 3.9$ & $68.6 \pm 4.0$ & $0.31 \pm 0.1$ \\
Hip measurement $(\mathrm{cm})$ & $99.8 \pm 4.7$ & $97.7 \pm 4.6$ & $2.06 \pm 0.6^{*}$ & $100 \pm 2.6$ & $100.1 \pm 2.6$ & $0.31 \pm 0.1^{* *}$ \\
SBP $(\mathrm{mmHg})$ & $120.5 \pm 1.6$ & $117.8 \pm 2.5$ & $2.5 \pm 2.9$ & $110.6 \pm 3.4$ & $108.1 \pm 1$ & $2.6 \pm 1.4$ \\
DBP (mmHg) & $75.3 \pm 1.0$ & $72 \pm 2.5$ & $3.1 \pm 3.1^{*}$ & $70.6 \pm 2.6$ & $67.5 \pm 1.7$ & $3.3 \pm 1.8$ \\
HR (beats/min) & $84.8 \pm 1.6$ & $81.2 \pm 2.2$ & $3.62 \pm 2.7$ & $84.5 \pm 2.0$ & $88.1 \pm 2.8$ & $3.5 \pm 1.7$ \\
RR (breaths/min) & $22.5 \pm 0.8$ & $21.5 \pm 0.9$ & $1.0 \pm 0.4^{*}$ & $21.6 \pm 0.7$ & $21.4 \pm 0.7$ & $0.2 \pm 0.1$ \\
$\mathrm{MaxVO}_{2}(\mathrm{ml} / \mathrm{kg} / \mathrm{min})$ & $587.9 \pm 21.7$ & $664.9 \pm 23.1$ & $76.1 \pm 10.8^{*}$ & $588.3 \pm 41.1$ & $591.7 \pm 36$ & $3.4 \pm 1.3^{* *}$ \\
\hline
\end{tabular}

Results are presented as the mean \pm standard error of the mean.

$*_{p}<0.05$, between baseline and after exercise values, by Mann-Whitney U test

** $p<0.05$, between difference scores of training and control groups, by Mann-Whitney U test

BMI: body mass index; DBP: diastolic blood pressure; HR: heart rate; $\max \mathrm{VO}_{2}$ : maximal oxygen consumption; SBP: systolic blood pressure; RR: respiratory rate

using the International Physical Activity Questionnaire ${ }^{14)}$. The exercise program included the following: 1. Warm-up and cool-down exercises: Walking on a treadmill at a low pace. 2. Aerobic exercise: A step was used for aerobic training. All patients started training with a $10 \mathrm{~cm}$ high step. Five weeks later, it was raised to a height of $15-20 \mathrm{~cm}$. Depending on the exercise tolerance of the patient, the exercise duration was increased from 5-7 min to $20 \mathrm{~min}$ over the 8 weeks of the study. The exercise was submaximal in intensity. The Borg Scale was used to rate perceived exertion $^{15)}$. Daily exercise was considered 1 session, and the session intensity was equivalent to $10-15$ points on the Borg Scale, thus achieving $65-70 \%$ of the maximum heart rate according to the American College of Sports Medicine. 3. Resistance exercise: Isoflex exercises utilizing an elastic band targeted the following muscles: Hip flexors, extensors, abductors, adductors, hamstrings, internal and external obliques, rectus abdominis, back extensors, scapular, and quadriceps muscles. The participants performed 15 repetitions of each exercise, with a minimum resting period of $30 \mathrm{~s}$ and a maximum of 1 min between each repetition. Exercise intensity was controlled individually, with the patient rating the exercise intensity according to a perceived exertion scale for resistance exercise ${ }^{16}$. The patients were advised to maintain an exercise intensity close to 5 or 6 , which corresponded to a perception of "somewhat intense" exercise.

Statistical tests were performed using the SPSS (Statistical Package for the Social Sciences for Windows, version 15.0, SPSS, Chicago, IL, USA). The normal distribution of the variables was determined using the KolmogorovSmirnov test. Only the BMI and FSH/LH variables were distributed normally. Due to the small sample size and multiple non-normally distributed variables, differences between the training and control groups were assessed using the MannWhitney $U$ test. The pre- and post-intervention values of the groups were compared using the Wilcoxon signed rank test. The results are presented as the mean \pm standard error of the mean. A p value $<0.05$ was considered statistically significant.

\section{RESULTS}

During the 3 year period, 382 patients were identified as having PCOS. Of these, 56 fulfilled the inclusion criteria. The 32 patients who agreed to participate in the study were divided randomly into groups of 16 patients each. However, two patients in the training group were eventually excluded because of inadequate attendance $(<75 \%$ of the training sessions). Thus, the study was completed by 14 patients in the training group and 16 in the control group. All 30 patients fulfilled the Rotterdam Criteria for PCOS: 30 had polycystic ovaries and anovulation, $26(86.7 \%)$ had clinical hyperandrogenism, and $19(63.3 \%)$ had biochemical hyperandrogenism. The mean age of the patients was 24.45 \pm 2.8 years (range: $17-34$ years).

The anthropometric and cardiovascular parameters of the two groups are listed in Table 1, and the metabolic parameters are shown in Table 2. Patients in the control and training groups did not significantly differ in their baseline parameters. In the training group, several anthropometric and cardiovascular parameters improved significantly after the exercise program; they showed significant decreases in waist circumference $(\mathrm{p}=0.02)$, hip circumference $(\mathrm{p}=$ $0.01)$, diastolic blood pressure $(\mathrm{p}=0.04)$, and respiratory rate $(\mathrm{p}=0.04)$ and a significant increase in $\mathrm{MaxVO}_{2}(\mathrm{p}=$ 0.001 ). In the control group, anthropometric and cardiovascular parameters were not significantly different after 8 weeks compared to the baseline values. The differences in hip circumference $(\mathrm{p}=0.04)$ and $\mathrm{MaxVO}_{2}(\mathrm{p}<0.001)$ after 8 weeks were significantly higher in the training group than in controls (Table 1).

The changes in hormone levels and metabolic parameters between the two groups are shown in Table 2. In the training group, the decreases in LDL cholesterol $(\mathrm{p}<0.001)$, total cholesterol $(p=0.01)$, fasting glucose $(p<0.001)$, fasting insulin $(\mathrm{p}=0.001)$, and HOMA $(\mathrm{p}<0.001)$ after exercise were significant, as was the increase in $\operatorname{HDL}(\mathrm{p}=0.002)$. In controls, none of the hormonal or metabolic parameters were significantly different at the end of the study period. The differences in LDL cholesterol $(\mathrm{p}=0.01)$, HDL $(\mathrm{p}=$ 
Table 2. Comparison of hormone levels and metabolic parameters between and within groups

\begin{tabular}{lcccccc}
\hline & \multicolumn{3}{c}{ Training group $(\mathrm{n}=14)$} & \multicolumn{3}{c}{ Control group $(\mathrm{n}=16)$} \\
\hline Variable & Baseline & 8 w later & Difference & Baseline & 8 w later & Difference \\
\hline FSH $(\mathrm{IU} / \mathrm{L})$ & $5.4 \pm 0.23$ & $5.0 \pm 0.3$ & $0.4 \pm 0.2$ & $5.4 \pm 0.2$ & $5.3 \pm 0.2$ & $0.04 \pm 0.08$ \\
LH $(\mathrm{IU} / \mathrm{L})$ & $7.1 \pm 1.5$ & $8.8 \pm 1.57$ & $1.7 \pm 0.9$ & $12.5 \pm 1.3$ & $12.4 \pm 1.2$ & $0.1 \pm 0.1$ \\
FSH/LH & $1.0 \pm 0.1$ & $1.0 \pm 0.2$ & $0.02 \pm 0.2$ & $0.5 \pm 0.04$ & $0.5 \pm 0.05$ & $0.0 \pm 0.0$ \\
$\mathrm{E}_{2}$ (pmol/L) & $36.0 \pm 8.4$ & $63.5 \pm 14$ & $27.4 \pm 18.5$ & $56.7 \pm 3.8$ & $56.8 \pm 3.8$ & $0.12 \pm 0.1$ \\
Total testosterone $(\mathrm{nmol} / \mathrm{L})$ & $1.2 \pm 0.9$ & $1.1 \pm 0.9$ & $0.1 \pm 0.7$ & $1.2 \pm 0.1$ & $1.2 \pm 0.2$ & $0.2 \pm 0.7$ \\
Free testosterone $(\mathrm{pg} / \mathrm{ml})$ & $3.15 \pm 0.0$ & $3.15 \pm 0.1$ & $0.00 \pm 0.0$ & $2.9 \pm 0.1$ & $2.83 \pm 0.2$ & $0.10 \pm 0.1$ \\
HDL-C $(\mathrm{mg} / \mathrm{dl})$ & $44.6 \pm 0.63$ & $45.2 \pm 0.6$ & $0.6 \pm 0.1^{*}$ & $46.1 \pm 0.9$ & $46.1 \pm 1$ & $0.0 \pm 0.5$ \\
LDL-C $(\mathrm{mg} / \mathrm{dl})$ & $122.6 \pm 6.1$ & $114 \pm 4.9$ & $8.62 \pm 1.5^{*}$ & $118.5 \pm 3.2$ & $118.6 \pm 3.2$ & $0.1 \pm 0.2^{* *}$ \\
Triglycerides $(\mathrm{mg} / \mathrm{dl})$ & $141.1 \pm 0.5$ & $138.2 \pm 0.8$ & $2.8 \pm 1.3$ & $139.6 \pm 2.2$ & $140.5 \pm 1.9$ & $0.8 \pm 0.4^{* *}$ \\
Total Cholesterol $(\mathrm{mg} / \mathrm{dl})$ & $198.6 \pm 3.7$ & $195.1 \pm 3$ & $3.4 \pm 1.1^{*}$ & $200.5 \pm 4.1$ & $200.7 \pm 4.0$ & $0.2 \pm 0.7^{* *}$ \\
Fasting glucose $(\mathrm{mg} / \mathrm{dl})$ & $98.1 \pm 2.4$ & $93.6 \pm 2.3$ & $4.4 \pm 2.5^{*}$ & $97.7 \pm 2.0$ & $97.3 \pm 2.3$ & $0.4 \pm 0.3^{* *}$ \\
Fasting insulin $(\mu \mathrm{U} / \mathrm{ml})$ & $14.7 \pm 1$ & $13.9 \pm 1$ & $0.8 \pm 0.1^{*}$ & $14.3 \pm 1$ & $14.5 \pm 0.8$ & $0.2 \pm 0.2^{* *}$ \\
HOMA index & $3.2 \pm 0.3$ & $2.9 \pm 0.2$ & $0.3 \pm 0.0^{*}$ & $3.1 \pm 0.2$ & $3.1 \pm 0.2$ & $0.1 \pm 0.1^{* *}$ \\
\hline
\end{tabular}

Results are presented as the mean \pm standard error of mean

${ }^{*} \mathrm{p}<0.05$, between baseline and after exercise values, by Mann-Whitney U test

$* * \mathrm{p}<0.05$, between difference scores of training and control groups, by Mann-Whitney U test

$\mathrm{E}_{2}$ : estradiol; FSH: follicle stimulating hormone; HDL-C: high-density lipoprotein cholesterol; HOMA: homeostatic model assessment; LDL-C: low-density lipoprotein cholesterol; LH: luteinizing hormone

$0.05)$, triglycerides $(p=0.005)$, total cholesterol $(p=0.01)$, fasting glucose $(p=0.01)$, fasting insulin $(p=0.001)$, and HOMA index $(\mathrm{p}<0.001)$ values were significantly greater in the training group than in controls at the end of the study (Table 2). The mean menstrual cycle interval did not change significantly in the control group $(46.12 \pm 1.3$ days vs. 47.05 \pm 1.2 days, $\mathrm{p}=0.125$ ) but decreased significantly in the training group after exercise (48.09 days vs. 27.3 days, $\mathrm{p}=$ $0.04)$.

\section{DISCUSSION}

We investigated the effects of an 8-week structured exercise program on non-overweight patients with PCOS. Many studies have reported that exercise training has beneficial effects on cardiopulmonary functional capacity and metabolic syndrome parameters in overweight patients with PCOS; however, this is the first study to evaluate the benefits of structured exercise in non-overweight PCOS patients. We found that 8 weeks of structured exercise was effective for improving anthropometric, cardiovascular, and metabolic parameters and for regulating menstrual cycles in non-overweight patients with PCOS, despite the absence of significant changes in sex hormones.

The beneficial effects of physical fitness are related to its reduction of the risk of cardiovascular dysfunction and to the delayed onset of metabolic syndrome activity of the proteins involved in insulin signal transduction in skeletal muscles ${ }^{17-19)}$. Therefore, by following a systematic exercise program, PCOS patients can improve their cardiopulmonary functional capacity and insulin sensitivity. Modalities other than fitness have been suggested for the treatment of PCOS. Fux Otta et al. ${ }^{20)}$ reported the additive effect of metformin on diet and exercise in the improvement of hyperandrogenism and insulin resistance. Although some studies have reported additive benefits of insulin sensitizers ${ }^{21,22)}$, others have not $^{23}$, 24). In contrast, all studies of PCOS have found that lifestyle changes should be considered in the management of these patients. However, there is no consensus on the basic variables of exercise training, including its type, intensity, duration, frequency, and progression. Orio et al. ${ }^{25}$ ) evaluated whether the effects of a training program were maintained after its cessation. They found that 12 weeks of non-training resulted in a complete loss of all favorable adaptations obtained from the exercise program. Therefore, exercise may need to be maintained throughout life for its benefits to be preserved. Although other studies have evaluated the impact of diet on the treatment of PCOS, we found that structured exercise alone was beneficial, at least in the short-term.

The role of non-pharmacological therapy in PCOS patients has been evaluated in several studies. Giallauria et al. $^{26)}$ assigned 124 PCOS patients to 2 groups for a 3-month exercise program. They demonstrated that exercise improved autonomic function and inflammatory patterns. In a similar study (based on 90 young women with PCOS who were randomly divided into 2 groups ${ }^{27)}$ ), patients who participated in a 3-month structured exercise program exhibited improvements in cardiopulmonary functional capacity. Other studies have reported greater reductions in BMI than our study ${ }^{28)}$, but we did not focus on weight loss, as the patients were not overweight. Nevertheless, they experienced important changes in several cardiovascular parameters and in $\mathrm{MaxVO}_{2}$.

Among the PCOS studies in the literature, positive results have been obtained in overweight patients who exercised for 12-24 weeks ${ }^{26-28)}$. Exactly when the beneficial effects of exercise can first be observed has not been determined. We found that in non-overweight PCOS patients with adequate $(\geq 75 \%$ ) participation in an exercise program consisting of aerobic and resistance exercise, anthropometric, metabolic, 
and cardiovascular parameters were already improved after 8 weeks. However, whether the same effects occur in morbidly obese patients with PCOS or whether they are apparent after a longer or shorter period of exercise remains to be determined.

Several studies have reported that diet changes, either alone or in combination with exercise, resulting in a weight loss of $5-10 \%$ could restore menstrual cycles. Weight reduction also has been shown to improve both the reproductive and the metabolic features of PCOS. Weight loss normalizes ovulation, ameliorates hyperandrogenism, and improves insulin sensitivity ${ }^{29)}$. In the present study, structured exercise for 8 weeks was sufficient for non-overweight patients with PCOS to achieve significant improvements in waist and hip measurements but did not lead to significant weight loss.

Our results suggest that an 8 -week exercise program improves reproductive and metabolic disorders related to metabolic syndrome in non-overweight PCOS patients. Long-term regular exercise may provide better results in metabolic parameters and improve the quality of life of these patients. The effects of exercise may occur more quickly in non-overweight than in overweight PCOS patients. The duration of exercise that achieves the maximum benefits may differ between these two groups of patients and requires further study.

\section{REFERENCES}

1) Ehrmann DA: Polycystic ovary syndrome. N Engl J Med, 2005, 352: 1223 1236. [Medline] [CrossRef]

2) Sheehan MT: Polycystic ovarian syndrome: diagnosis and management Clin Med Res, 2004, 2: 13-27. [Medline] [CrossRef]

3) Diamanti-Kandarakis E, Papavassiliou AG, Kandarakis SA, et al.: Pathophysiology and types of dyslipidemia in PCOS. Trends Endocrinol Metab, 2007, 18: 280-285. [Medline] [CrossRef]

4) Moran LJ, Lombard CB, Lim S, et al.: Polycystic ovary syndrome and weight management. Womens Health (Lond Engl), 2010, 6: 271-283. [Medline] [CrossRef]

5) Thomson RL, Buckley JD, Noakes M, et al.: The effect of a hypocaloric diet with and without exercise training on body composition, cardiometabolic risk profile, and reproductive function in overweight and obese women with polycystic ovary syndrome. J Clin Endocrinol Metab, 2008 93: 3373-3380. [Medline] [CrossRef]

6) Lee HC, Heo T: Effects of exercise therapy on blood lipids of obese women. J Phys Ther Sci, 2014, 26: 1675-1677. [Medline] [CrossRef]

7) Tamura T, Kida K, Seki T, et al.: Study of the relationship between exercise therapy and diet therapy in type 2 diabetes mellitus patients. J Phys Ther Sci, 2011, 23: 485-488. [CrossRef]

8) Li L, Chen X, He Z, et al.: Clinical and metabolic features of polycystic ovary syndrome among Chinese adolescents. J Pediatr Adolesc Gynecol, 2012, 25: 390-395. [Medline] [CrossRef]

9) Thomson RL, Buckley JD, Moran LJ, et al.: Comparison of aerobic exercise capacity and muscle strength in overweight women with and without polycystic ovary syndrome. BJOG, 2009, 116: 1242-1250. [Medline] [CrossRef]

10) Rotterdam ESHRE/ASRM-Sponsored PCOS Consensus Workshop
Group: Revised 2003 consensus on diagnostic criteria and long-term health risks related to polycystic ovary syndrome. Fertil Steril, 2004, 81: 19-25. [CrossRef]

11) Carmina E, Orio F, Palomba S, et al.: Ovarian size and blood flow in women with polycystic ovary syndrome and their correlations with endocrine parameters. Fertil Steril, 2005, 84: 413-419. [Medline] [CrossRef]

12) Ferriman D, Gallwey JD: Clinical assessment of body hair growth in women. J Clin Endocrinol Metab, 1961, 21: 1440-1447. [Medline] [CrossRef]

13) Kervio G, Carre F, Ville NS: Reliability and intensity of the six-minute walk test in healthy elderly subjects. Med Sci Sports Exerc, 2003, 35: 169174. [Medline] [CrossRef]

14) Saglam M, Arikan $H$, Savci $S$, et al.: International physical activity questionnaire: reliability and validity of the Turkish version. Percept Mot Skills, 2010, 111: 278-284. [Medline] [CrossRef]

15) Borg G: Perceived exertion as an indicator of somatic stress. Scand J Rehabil Med, 1970, 2: 92-98. [Medline]

16) Robertson RJ, Goss FL, Rutkowski J, et al: Concurrent validation of the OMNI perceived exertion scale for resistance exercise. Med Sci Sports Exerc, 2003, 35: 333-341. [Medline] [CrossRef]

17) Lee Y, Jun I, Ju S: Impact of home exercise training on patients with acute myocardial infarction. J Phys Ther Sci, 2012, 24: 743-745. [CrossRef]

18) Kim DY, Seo BD, Kim DJ: Effect of walking exercise on changes in cardiorespiratory fitness, metabolic syndrome markers, and high-molecularweight adiponectin in obese middle-aged women. J Phys Ther Sci, 2014, 26: 1723-1727. [Medline] [CrossRef]

19) Yagura $C$, Takamura N, Goto $Y$, et al.: Cardiorespiratory fitness and metabolic markers in healthy young adult men. J Phys Ther Sci, 2011, 23: 845849. [CrossRef]

20) Fux Otta C, Wior M, Iraci GS, et al.: Clinical, metabolic, and endocrine parameters in response to metformin and lifestyle intervention in women with polycystic ovary syndrome: a randomized, double-blind, and placebo control trial. Gynecol Endocrinol, 2010, 26: 173-178. [Medline] [CrossRef]

21) Harborne L, Fleming R, Lyall H, et al.: Descriptive review of the evidence for the use of metformin in polycystic ovary syndrome. Lancet, 2003, 361: 1894-1901. [Medline] [CrossRef]

22) Nestler JE: Metformin for the treatment of the polycystic ovary syndrome. N Engl J Med, 2008, 358: 47-54. [Medline] [CrossRef]

23) Açbay $\mathrm{O}$, Gündoğdu S: Can metformin reduce insulin resistance in polycystic ovary syndrome? Fertil Steril, 1996, 65: 946-949. [Medline]

24) Ehrmann DA, Cavaghan MK, Imperial J, et al.: Effects of metformin on insulin secretion, insulin action, and ovarian steroidogenesis in women with polycystic ovary syndrome. J Clin Endocrinol Metab, 1997, 82: 524-530. [Medline]

25) Orio F, Giallauria F, Palomba S, et al.: Metabolic and cardiopulmonary effects of detraining after a structured exercise training programme in young PCOS women. Clin Endocrinol (Oxf), 2008, 68: 976-981. [Medline] [CrossRef]

26) Giallauria F, Palomba S, Maresca L, et al.: Exercise training improves autonomic function and inflammatory pattern in women with polycystic ovary syndrome (PCOS). Clin Endocrinol (Oxf), 2008, 69: 792-798. [Medline] [CrossRef]

27) Vigorito $\mathrm{C}$, Giallauria F, Palomba S, et al.: Beneficial effects of a threemonth structured exercise training program on cardiopulmonary functional capacity in young women with polycystic ovary syndrome. J Clin Endocrinol Metab, 2007, 92: 1379-1384. [Medline] [CrossRef]

28) Liao LM, Nesic J, Chadwick PM, et al.: Exercise and body image distress in overweight and obese women with polycystic ovary syndrome: a pilot investigation. Gynecol Endocrinol, 2008, 24: 555-561. [Medline] [CrossRef]

29) Panidis D, Farmakiotis D, Rousso D, et al.: Obesity, weight loss, and the polycystic ovary syndrome: effect of treatment with diet and orlistat for 24 weeks on insulin resistance and androgen levels. Fertil Steril, 2008, 89: 899-906. [Medline] [CrossRef] 